\title{
THE ELECTROMAGNETIC AND PROCA FIELDS REVISITED: A UNIFIED QUANTIZATION ⿵
}

\author{
Víctor Aldaya ${ }^{1,2}$, Manuel Calixto ${ }^{1,5}$ and Miguel Navarro ${ }^{3,4}$
}

1. Instituto Carlos I de Física Teórica y Computacional, Facultad de Ciencias, Universidad de Granada, Campus de Fuentenueva, 18002, Granada, Spain.

2. IFIC, Centro Mixto Universidad de Valencia-CSIC, Burjassot 46100-Valencia, Spain.

3. The Blackett Laboratory, Imperial College, Prince Consort Road, London SW7 2BZ; United Kingdom.

4. Instituto de Matemáticas y Física Fundamental, CSIC, Serrano 113-123, 28006 Madrid, Spain

5. Departamento de Física Teórica y del Cosmos, Facultad de Ciencias, Universidad de Granada, Campus de Fuentenueva, Granada 18002, Spain

\begin{abstract}
Quantizing the electromagnetic field with a group formalism faces the difficulty of how to turn the traditional gauge transformation of the vector potential, $A_{\mu}(x) \rightarrow A_{\mu}(x)+\partial_{\mu} \varphi(x)$, into a group law. In this paper it is shown that the problem can be solved by looking at gauge transformations in a slightly different manner which, in addition, does not require introducing any BRST-like parameter. This gauge transformation does not appear explicitly in the group law of the symmetry but rather as the trajectories associated with generalized equations of motion generated by vector fields with null Noether invariants. In the new approach the parameters of the local group, $U(1)(\vec{x}, t)$, acquire dynamical content outside the photon mass shell, a fact which also allows a unified quantization of both the electromagnetic and Proca fields.
\end{abstract}

*Work partially supported by the Comisión Interministerial de Ciencia y Tecnología. 


\section{Introduction}

According to the minimal interaction principle, in order to extend any internal symmetry of the matter fields to the gauge level, i.e. turning the group parameters $\varphi^{a}$ into functions on configuration space, a vector potential $A_{\mu}^{a}(x)$ which transforms as a connection form must be introduced. For the particular case of the $U(1)$-symmetry this vector potential acquires only a derivative of the gauge parameter under a gauge transformation:

$$
A_{\mu}(x) \rightarrow A_{\mu}(x)+\partial_{\mu} \varphi(x) .
$$

This is the standard way of introducing the electromagnetic interaction in a geometric formulation. However, the electromagnetic field has its own entity, not necessarily attached to the non-tensorial part of the gauge transformations, and its quantization can be achieved directly in terms of the field strengths [1]. Quantizing in terms of $A_{\mu}$ results in a redundant system which must be further constrained by the so-called Gauss law. Let us briefly review the quantum origin of this constraint.

Maxwell's theory for the electromagnetic field without sources may be derived from the Lagrangian density:

$$
\mathcal{L}=-\frac{1}{4} F^{\mu \nu} F_{\mu \nu}=\frac{1}{2}\left(\vec{E}^{2}-\vec{B}^{2}\right)
$$

where

$$
F_{\mu \nu} \equiv \partial_{\mu} A_{\nu}-\partial_{\nu} A_{\mu}, \quad \vec{E} \equiv-\dot{\vec{A}}-\vec{\nabla} A^{0}, \quad \vec{B} \equiv \nabla \times \vec{A}
$$

are the electromagnetic tensor and the electric and magnetic fields, respectively. The equations of motion are:

$$
\partial_{\mu} F^{\mu \nu}=0
$$

for which the time and space components are the Gauss law and the Ampère law, respectively:

$$
\nabla \cdot \vec{E}=0, \quad \dot{\vec{E}}=\nabla \times \vec{B}
$$

The canonical quantization of this system encounters a problem when submitted to a Hamiltonian description, due to the fact that the Lagrangian (2) does not depend on $\dot{A}^{0}$, so that there is no momentum conjugate to $A^{0}$. In other words, this Lagrangian is not regular. This means, in general, that not all Lagrangian equations can be written in Hamiltonian form and some must be added to the set of Hamilton equations as constraints. This is precisely the case of the Gauss law when one uses the gauge freedom to set the non-covariant condition $A^{0}=0$, i.e. the so-called "Weyl gauge" (see e.g. [2]).

In a previous paper [3] the quantization of the free electromagnetic field was achieved in such a way that the Gauss law appeared on the same footing as 
the ordinary Hamilton equations of motion and not as a constraint. The algorithm used was a group approach to quantization (GAQ) [4, 5] formulated on an infinite-dimensional Lie group parameterized by the strengths $\vec{E}(x)$ and $\vec{B}(x)$, as well as the time variable. However, the analogous quantization of the free electromagnetic system in terms of the vector potential $A_{\mu}$ found it difficult to accommodate the gauge transformation property (1) to a group law. The introduction of an extra group parameter, which revealed itself as being some sort of bosonic BRST parameter [6], was apparently needed.

In this paper we propose a new infinite-dimensional Lie group $\tilde{G}$, with a principal bundle structure $\tilde{G} \rightarrow \tilde{G} / T$, parameterized, roughly speaking, by $A_{\mu}(\vec{x}, t)$, the Poincaré variables and the coordinates of the local $U(1)(\vec{x}, t)$, which plays the role of the structure group $T$. This subgroup generalizes the standard $U(1)$-phase invariance in Quantum Mechanics, so that the extra equivariance conditions associated with the local subgroup $U(1)(\vec{x}, t)$ will provide the traditional constraints of the theory. The construction of the new group law has required a review of the concepts of gauge symmetry and constraints and has led, as a byproduct, to a unified quantization of both the electromagnetic and Proca fields, within the same general scheme of quantization based on a group (GAQ).

Let us motivate the explicit group law to be proposed. Going back to the Lagrangian analysis of the origin of the constraints outlined above, we must note that the non-regularity property of the Lagrangian (2) can be covariantly expressed by stating that $\mathcal{L}$ does not depend on the covariant quantity $\Phi \equiv \partial_{\mu} A^{\mu}$. This suggests resorting to the new, regular Lagrangian (the Fermi Lagrangian if $\lambda=1)$

$$
\mathcal{L}^{\prime}=-\frac{1}{4} F^{\mu \nu} F_{\mu \nu}-\frac{\lambda}{2}\left(\partial_{\mu} A^{\mu}\right)^{2}
$$

for arbitrary $\lambda$, which keeps a residual (covariant) gauge invariance $A_{\mu}(x) \rightarrow$ $A_{\mu}(x)+\partial_{\mu} \varphi(x)$ under (on-shell) functions $\varphi(x)$ such that $\partial_{\mu} \partial^{\mu} \varphi(x)=0$. With this residual symmetry, we could formally associate a Noether charge of the form

$$
Q_{\varphi}=\int d^{3} x \frac{\partial \mathcal{L}^{\prime}}{\partial \dot{A}_{\mu}} \partial_{\mu} \varphi=\lambda \int d^{3} x(\dot{\Phi} \varphi-\Phi \dot{\varphi}),
$$

where we have used the equations of motion:

$$
\partial_{\mu} F^{\mu \nu}+\lambda \partial^{\nu} \Phi=0 \Rightarrow \partial_{\mu} \partial^{\mu} \Phi=0 .
$$

The standard Maxwell's equations (4) can be regained simply by putting $\Phi=$ constant as a (classical) constraint. In particular, the constraint $\dot{\Phi}=0$ in (8) reproduces the Gauss Law.

A glance at eq. (7) reveals that the quantity $\dot{\Phi}$ behaves as a generator of gauge transformations (those depending only on the Cauchy hypersurface parameters; see [2] for the non-covariant case). In fact, both $\Phi$ and $\dot{\Phi}$ close a Poisson algebra 
with the rest of the dynamical variables and the Hamiltonian associated with $\mathcal{L}^{\prime}$. It is straightforward to compute the following Poisson brackets $(\lambda=1$ m):

$$
\begin{array}{ll}
\left\{A_{\mu}(\vec{x}, t), A_{\nu}(\vec{y}, t)\right\}=0 & \left\{\dot{A}_{\mu}(\vec{x}, t), \dot{A}_{\nu}(\vec{y}, t)\right\}=0 \\
\left\{\dot{A}_{\mu}(\vec{x}, t), A^{\nu}(\vec{y}, t)\right\}=g_{\mu}^{\nu} \delta^{3}(\vec{x}-\vec{y}) & \{\dot{\Phi}(\vec{x}, t), \Phi(\vec{y}, t)\}=0 \\
\left\{\Phi(\vec{x}, t), A^{\mu}(\vec{y}, t)\right\}=g_{0}^{\mu} \delta^{3}(\vec{x}-\vec{y}) & \left\{\Phi(\vec{x}, t), \dot{A}^{\mu}(\vec{y}, t)\right\}=-g_{i}^{\mu} \partial^{i} \delta^{3}(\vec{x}-\vec{y}) \\
\left\{\dot{\Phi}(\vec{x}, t), A^{\mu}(\vec{y}, t)\right\}=g_{i}^{\mu} \partial^{i} \delta^{3}(\vec{x}-\vec{y}) & \left\{\dot{\Phi}(\vec{x}, t), \dot{A}^{\mu}(\vec{y}, t)\right\}=-g_{0}^{\mu} \nabla^{2} \delta^{3}(\vec{x}-\vec{y}) \\
\left\{A_{\mu}, H\right\}=\dot{A}_{\mu} & \left\{\dot{A}_{\mu}, H\right\}=\nabla^{2} A_{\mu} \\
\{\Phi, H\}=\Phi & \{\dot{\Phi}, H\}=\nabla^{2} \Phi .
\end{array}
$$

The statement that the Maxwell's tensor is gauge invariant, can now be expressed by: $\left\{F_{\mu \nu}(\vec{x}, t), \Phi(\vec{y}, t)\right\}=\left\{F_{\mu \nu}(\vec{x}, t), \dot{\Phi}(\vec{y}, t)\right\}=0$, as it can be easily derived from (9).

As a Lie algebra, (9) is a central extension by $U(1)$ characterized by a (Lie algebra) co-cycle involving the generators $(A, \dot{A}, \Phi, \dot{\Phi})$, much in the same way the Poisson bracket $\{p, q\}=1$ characterizes the (Lie algebra) co-cycle of the Heisenberg-Weyl subalgebra for a particle in particle Mechanics. From this co-

cycle we can read immediately that the couple $(A, \dot{A})$ corresponds to a canonicallyconjugate pair of variables and that the $(\Phi, \dot{\Phi})$ variables are not completely devoid of dynamical content, as the bracket $\{\dot{\Phi}(\vec{x}, t), \Phi(\vec{y}, t)\}=0$ might suggest at first sight, due to the non-diagonal terms in the co-cycle (lines $3^{\text {rd }}$ and $4^{\text {th }}$ in (9)). For this reason, the variables $\Phi, \dot{\Phi}$ cannot be properly associated with the usual null Noether charge gauge generators. Rather, these variables will be considered simply as the generators of the structure group $T$ and related with the constraints.

As our starting point to perform the quantization of the electromagnetic and Proca fields, we shall adopt the abstract structure of the Poisson algebra above (with the Poincaré generators added), but we shall deform it with a non-trivial central term of the form $\{\dot{\Phi}(\vec{x}, t), \Phi(\vec{y}, t)\}=m^{2} \delta^{3}(\vec{x}-\vec{y})$ ( $m$ being a parameter with mass dimension; we are using natural unities, $\hbar=1=c$ ) and construct the group law by standard exponentiation. The new central term, parameterized by $m$, modifies the dynamical content of the group variables, transferring degrees of freedom between the $A$ and $\Phi$ variables. In the case $m \neq 0$ the group co-cycle diagonalizes in a new set of variables which correspond to the Proca field and some sort of scalar field.

\section{Unified quantization of the electromagnetic and Proca fields}

According to the general prescription of GAQ we shall start from a group law, which is inspired in the (classical) Poisson algebra (9), involving the Poincaré pa-

*We adopt this choice for simplicity, but the results are $\lambda$ independent. 
rameters $x^{\mu}, \Lambda_{\nu}^{\mu}$, the Fourier coefficients $a_{\mu}(k), a_{\mu}^{+}(k)$ of the field $A_{\mu}$, the Fourier coefficients $\phi(k), \phi^{+}(k)$ of the local $U(1)_{l o c}$ subgroup and the parameter $\zeta$ associated with the central $U(1)$ subgroup. The entire group $\tilde{G}$ will be regarded either as: a) a non-central extension by a group $T$ (parameterized by $\phi(k), \phi^{+}(k)$ and $\zeta$ ) of the group constituted by the space-time symmetry and the Fourier coefficients of $A_{\mu}$, or b) a central extension by $U(1)$ of the remainder: $G \equiv \tilde{G} / U(1)$. In both cases the group law corresponding to the central parameter will be characterized by a set of cocycles $\xi_{1}, \xi_{2}, \xi_{3}$ (see below) defined on the whole "classical" group $G$. As mentioned above, the equations associated with the $T$-equivariance condition, other than the usual $U(1)$ condition, are interpreted as constraints in the general group-quantization formalism [5].

The precise group law $g^{\prime \prime}=g^{\prime} * g$ is:

$$
\begin{aligned}
a_{\mu}^{\prime \prime}(k)= & a_{\mu}^{\prime}(k) \exp \left(-i k \cdot \Lambda^{\prime} x\right)+\Lambda_{\mu}^{\prime \nu} a_{\nu}\left(\Lambda^{-1 \prime} k\right) \\
a_{\mu}^{+\prime \prime}(k)= & a_{\mu}^{+\prime}(k) \exp \left(i k \cdot \Lambda^{\prime} x\right)+\Lambda_{\mu}^{\prime \nu} a_{\mu}^{+}\left(\Lambda^{-1 \prime} k\right) \\
\phi^{\prime \prime}(k)= & \phi^{\prime}(k) \exp \left(-i k \cdot \Lambda^{\prime} x\right)+\phi\left(\Lambda^{-1 \prime} k\right) \\
\phi^{+\prime \prime}(k)= & \phi^{+\prime}(k) \exp \left(i k \cdot \Lambda^{\prime} x\right)+\phi^{+}\left(\Lambda^{-1 \prime} k\right) \\
x^{\prime \prime}= & x^{\prime}+\Lambda^{\prime} x \\
\Lambda^{\prime \prime}= & \Lambda^{\prime} \Lambda \\
\zeta^{\prime \prime}= & \zeta^{\prime} \zeta \exp \left[\frac{i}{2} \int \frac{d^{3} k}{2 k^{0}} i\left\{\xi_{1}\left(g^{\prime}, g\right)+\xi_{2}\left(g^{\prime}, g\right)+\xi_{3}\left(g^{\prime}, g\right)\right\}\right], \quad g, g^{\prime} \in G \\
\xi_{1}\left(g^{\prime}, g\right) \equiv & g^{\mu \sigma} \Lambda_{\sigma}^{\nu \prime}\left[a_{\mu}\left(\Lambda^{-1 \prime} k\right) a_{\nu}^{+\prime}(k) e^{i k \cdot \Lambda^{\prime} x}-a_{\mu}^{+}\left(\Lambda^{-1 \prime} k\right) a_{\nu}^{\prime}(k) e^{-i k \cdot \Lambda^{\prime} x}\right] \\
\xi_{2}\left(g^{\prime}, g\right) \equiv & i\left[\phi^{+\prime}(k)\left(\Lambda^{-1 \prime}\right)^{\mu \nu} k_{\nu} a_{\mu}\left(\Lambda^{-1 \prime} k\right) e^{i k \Lambda^{\prime} x}+\phi^{\prime}(k)\left(\Lambda^{-1 \prime}\right)^{\mu \nu} k_{\nu} a_{\mu}^{+}\left(\Lambda^{-1 \prime} k\right) e^{-i k \Lambda^{\prime} x}\right. \\
& \left.-k^{\mu} a_{\mu}^{+\prime}(k) \phi\left(\Lambda^{-1 \prime} k\right) e^{i k \cdot \Lambda^{\prime} x}-k^{\mu} a_{\mu}^{\prime}(k) \phi^{+}\left(\Lambda^{-1 \prime} k\right) e^{-i k \cdot \Lambda^{\prime} x}\right] \\
\xi_{3}\left(g^{\prime}, g\right) \equiv & k^{2}\left[\phi^{+\prime}(k) \phi\left(\Lambda^{-1 \prime} k\right) e^{i k \cdot \Lambda^{\prime} x}-\phi^{\prime}(k) \phi^{+}\left(\Lambda^{-1 \prime} k\right) e^{-i k \cdot \Lambda^{\prime} x}\right] .
\end{aligned}
$$

Note that, in fact, we are not actually dealing with the whole local group $U(1)_{l o c}$ but rather with a subgroup made of "on-shell" functions and related to the residual gauge invariance surviving in the Lagrangian (6); nevertheless, we shall refer to this symmetry simply as local $U(1)_{l o c}$ symmetry. A remarkable feature of the proposed group law is that, unlike in the standard formulation of gauge theories, the local $U(1)_{l o c}$ subgroup does not act on the vector potential parameters (as in Eq. (11)), thus obviating the need of introducing new (BRSTlike) parameters as in Ref. [6]. However, the local symmetry acts non-trivially on the $U(1)$ parameter $\zeta$, causing a change in the phase of the wave function (see below). Furthermore, the traditional transformation properties of the vector potential will appear as the trajectories of some of the generalized (quantum) equations of motion.

The arbitrariness in the choice of the value of $k^{2}$ in the co-cycle $\xi_{3}\left(g^{\prime}, g\right)$, extending the local $U(1)_{l o c}$ subgroup, will lead to a unified quantization of the 
electromagnetic field ( $k^{2}=0$ case) and the Proca field $\left(k^{2}=m^{2} \neq 0\right.$ case) when the $T$-equivariant conditions are imposed as constraints in both cases. We shall maintain $k^{2}$ throughout the following expressions, until distinction of the two cases becomes necessary.

From the group law (10), two sets of generators can be derived, the rightand left-invariant vector fields, out of which the physical operators of the theory and the polarization conditions, required to make the quantum representation irreducible, must be respectively constructed.

To save unnecessary calculations we shall discard the Lorentz subgroup, which plays no dynamical role, and simply comment on the unessential differences that could be obtained should we keep this subgroup. Under this simplification the two sets of generators are:

$$
\begin{aligned}
& \tilde{X}_{\zeta}^{R}=i \zeta \frac{\partial}{\partial \zeta} \equiv \Xi \\
& \tilde{X}_{a_{\mu}(k)}^{R}=e^{-i k x}\left[\frac{\delta}{\delta a_{\mu}(k)}-\frac{i}{2}\left[a^{\mu+}(k)+i \phi^{+}(k) k^{\mu}\right] \Xi\right] \\
& \tilde{X}_{a_{\mu}^{+}(k)}^{R}=e^{i k x}\left[\frac{\delta}{\delta a_{\mu}^{+}(k)}+\frac{i}{2}\left[a^{\mu}(k)-i \phi(k) k^{\mu}\right] \Xi\right] \\
& \tilde{X}_{\phi(k)}^{R}=e^{-i k x}\left[\frac{\delta}{\delta \phi(k)}-\frac{i}{2}\left[k^{2} \phi^{+}(k)-i k^{\mu} a_{\mu}^{+}(k)\right] \Xi\right] \\
& \tilde{X}_{\phi^{+}(k)}^{R}=e^{i k x}\left[\frac{\delta}{\delta \phi^{+}(k)}+\frac{i}{2}\left[k^{2} \phi(k)+i k^{\mu} a_{\mu}(k)\right] \Xi\right] \\
& \tilde{X}_{x^{\mu}}^{R}=\frac{\partial}{\partial x^{\mu}} \\
& \tilde{X}_{\zeta}^{L}=i \zeta \frac{\partial}{\partial \zeta} \equiv \Xi \\
& \tilde{X}_{a_{\mu}(k)}^{L}=\frac{\delta}{\delta a_{\mu}(k)}+\frac{i}{2}\left[a^{\mu+}(k)+i \phi^{+}(k) k^{\mu}\right] \Xi \\
& \tilde{X}_{a_{\mu}^{+}(k)}^{L}=\frac{\delta}{\delta a_{\mu}^{+}(k)}-\frac{i}{2}\left[a^{\mu}(k)-i \phi(k) k^{\mu}\right] \Xi \\
& \tilde{X}_{\phi(k)}^{L}=\frac{\delta}{\delta \phi(k)}+\frac{i}{2}\left[k^{2} \phi^{+}(k)-i k^{\mu} a_{\mu}^{+}(k)\right] \Xi \\
& \tilde{X}_{\phi^{+}(k)}^{L}=\frac{\delta}{\delta \phi^{+}(k)}-\frac{i}{2}\left[k^{2} \phi(k)+i k^{\mu} a_{\mu}(k)\right] \Xi \\
& \tilde{X}_{x^{\mu}}^{L}=\frac{\partial}{\partial x^{\mu}}-\int \frac{d^{3} k}{2 k^{0}} k^{\mu}\left[a_{\nu}(k) \frac{\delta}{\delta a_{\nu}(k)}-a_{\nu}^{+}(k) \frac{\delta}{\delta a_{\nu}^{+}(k)}\right] \\
& +\int \frac{d^{3} k}{2 k^{0}} k^{\mu}\left[\phi(k) \frac{\delta}{\delta \phi(k)}-\phi^{+}(k) \frac{\delta}{\delta \phi^{+}(k)}\right]
\end{aligned}
$$


From the left generators we obtain the following non-trivial commutation relations describing, in particular, the dynamical content of each parameter:

$$
\begin{array}{ll}
{\left[\tilde{X}_{x^{\mu}}^{L}, \tilde{X}_{a_{\nu}(k)}^{L}\right]=i k_{\mu} \tilde{X}_{a_{\nu}(k)}^{L}} & {\left[\tilde{X}_{x^{\mu}}^{L}, \tilde{X}_{a_{\nu}^{+}(k)}^{L}\right]=-i k_{\mu} \tilde{X}_{a_{\nu}^{+}(k)}^{L}} \\
{\left[\tilde{X}_{x^{\mu}}^{L}, \tilde{X}_{\phi(k)}^{L}\right]=i k_{\mu} \tilde{X}_{\phi(k)}^{L}} & {\left[\tilde{X}_{x^{\mu}}^{L}, \tilde{X}_{\phi^{+}(k)}^{L}\right]=-i k_{\mu} \tilde{X}_{\phi^{+}(k)}^{L}} \\
{\left[\tilde{X}_{a_{\mu}^{+}(k)}^{L}, \tilde{X}_{a_{\nu}\left(k^{\prime}\right)}^{L}\right]=i g^{\mu \nu} \Delta_{k k^{\prime}} \Xi} & {\left[\tilde{X}_{\phi^{+}(k)}^{L}, \tilde{X}_{\phi\left(k^{\prime}\right)}^{L}\right]=i k^{2} \Delta_{k k^{\prime}} \Xi} \\
{\left[\tilde{X}_{a_{\mu}(k)}^{L}, \tilde{X}_{\phi^{+}\left(k^{\prime}\right)}^{L}\right]=k^{\mu} \Delta_{k k^{\prime}} \Xi} & {\left[\tilde{X}_{a_{\mu}^{+}(k)}^{L}, \tilde{X}_{\phi\left(k^{\prime}\right)}^{L}\right]=k^{\mu} \Delta_{k k^{\prime}} \Xi,}
\end{array}
$$

where $\Delta_{k k^{\prime}}=2 k^{0} \delta^{3}\left(k-k^{\prime}\right)$ is the generalized delta function on the positive sheet of the mass hyperboloid. From the commutation relations (13), line 3, we observe that the four components of $a_{\nu}(k)$ and $a_{\nu}^{+}(k)$ are canonically conjugate variables $(\operatorname{det}(g) \neq 0)$ for any value of $k$, whereas the pair $\phi(k)$ and $\phi^{+}(k)$ are canonically conjugate out of the photon mass shell only. However, we also note that there is a piece of the Lie algebra cocycle, line 4 (corresponding to the group co-cycle $\xi_{2}\left(g^{\prime}, g\right)$ ), which mixes $a$ 's and $\phi$ 's. When the $U(1)_{l o c}$ parameters themselves acquire dynamical character, i.e. when the "photon" is off-shell, the cocycle can be diagonalized, thus transferring dynamical content from one of the pairs associated with the new electromagnetic coefficients to the new $U(1)_{l o c}$ parameters. In both cases there are four independent field degrees of freedom in the group $\tilde{G}$, but the $T$-equivariance condition (the constraints) will remove two degrees from the electromagnetic field in the case $k^{2}=0$, while in the case $k^{2}=m^{2}$ the $T$-equivariance condition will subtract only one degree of freedom, leaving three, which designate a massive vector field (see Sec. 2.2 for further discussion).

The GAQ formalism then continues finding the left-invariant 1-form $\Theta$ (the quantization form) associated with the central generator $\Xi$. The differential $d \Theta$ is a presymplectic form and its characteristic module $\operatorname{Ker} d \Theta \cap \operatorname{Ker} \Theta$, generates a left subalgebra $\mathcal{G}_{\Theta}$ called characteristic subalgebra (the kernel of the Lie algebra cocycle). The quotient $(\tilde{G}, \Theta) / \mathcal{G}_{\Theta}$ is a quantum manifold. For our case, they prove to be:

$$
\begin{aligned}
\Theta= & \frac{i}{2} \int \frac{d^{3} k}{2 k^{0}}\left\{g^{\mu \nu}\left(\left[a_{\mu}(k)-i k_{\mu} \phi(k)\right] d a_{\nu}^{+}(k)-\left[a_{\mu}^{+}(k)+i k_{\mu} \phi^{+}(k)\right] d a_{\nu}(k)\right]\right. \\
& +k_{\mu}\left[k_{\nu} \phi(k)+i a_{\nu}(k)\right] d \phi^{+}(k)-k_{\mu}\left[k_{\nu} \phi^{+}(k)-i a_{\nu}^{+}(k)\right] d \phi(k) \\
& \left.\left.+\left[a_{\mu}(k)-i k_{\mu} \phi(k)\right]\left[a_{\nu}^{+}(k)+i k_{\nu} \phi^{+}(k)\right] k^{\sigma} d x_{\sigma}\right)\right\}+\frac{d \zeta}{i \zeta} \\
\mathcal{G}_{\Theta}= & <\tilde{X}_{x^{\mu}}^{L}, \tilde{X}_{c(k)}^{L}, \tilde{X}_{c^{+}(k)}^{L}>\forall k
\end{aligned}
$$

where we have defined

$$
\tilde{X}_{c(k)}^{L} \equiv \tilde{X}_{\phi(k)}^{L}+i k_{\mu} \tilde{X}_{a_{\mu}(k)}^{L}, \quad \tilde{X}_{c^{+}(k)}^{L} \equiv \tilde{X}_{\phi^{+}(k)}^{L}-i k_{\mu} \tilde{X}_{a_{\mu}^{+}(k)}^{L} .
$$


We should note that $\mathcal{G}_{\Theta}$ would have included the Lorentz generators had we kept them in the theory. The vector fields in the characteristic subalgebra represent the generalized classical equations of motion. The invariant quantities under the above-mentioned generalized equations of motion, are the corresponding generalized Noether invariants defined as:

$$
F_{g_{j}} \equiv i_{\tilde{X}_{g_{j}}^{R}} \Theta, \quad \forall g_{j} \in \tilde{G} .
$$

For our case, they are:

$$
\begin{aligned}
F_{\phi(k)} & =i_{\tilde{X}_{\phi^{R}(k)}} \Theta=-i e^{-i k x} k^{\mu}\left[k_{\mu} \phi^{+}(k)-i a_{\mu}^{+}(k)\right] \equiv-i k^{2} \phi_{(0)}^{+}(k)-k^{\mu} a_{(0) \mu}^{+} \\
F_{\phi^{+}(k)} & =i_{\tilde{X}_{\phi^{+}(k)}^{R}} \Theta=i e^{i k x} k^{\mu}\left[k_{\mu} \phi(k)+i a_{\mu}(k)\right] \equiv i k^{2} \phi_{(0)}(k)-k^{\mu} a_{(0) \mu} \\
F_{a_{\mu}(k)} & =i_{\tilde{X}_{a_{\mu}(k)}^{R}} \Theta=e^{-i k x}\left[k^{\mu} \phi^{+}(k)-i a^{\mu+}(k)\right] \equiv k^{\mu} \phi_{(0)}^{+}(k)-i a_{(0)}^{\mu+}(k) \\
F_{a_{\mu}^{+}(k)} & =i_{\tilde{X}_{a_{\mu}^{R}(k)}^{R}} \Theta=e^{i k x}\left[k^{\mu} \phi(k)+i a^{\mu}(k)\right] \equiv k^{\mu} \phi_{(0)}(k)+i a_{(0)}^{\mu}(k) \\
F_{x^{\mu}} & =i_{\tilde{X}_{x^{R}}^{R}} \Theta=\int \frac{d^{3} k}{2 k^{0}} k_{\mu}\left[a_{(0)}^{\nu}(k)-i k^{\nu} \phi_{(0)}(k)\right]\left[a_{(0) \nu}^{+}(k)+i k_{\nu} \phi_{(0)}^{+}(k)\right],
\end{aligned}
$$

where $a_{(0) \mu}^{+}(k), a_{(0) \mu}(k), \phi_{(0)}^{+}(k), \phi_{(0)}(k)$ are the initial conditions.

Apart from the conventional evolution, generated by $\tilde{X}_{x^{\mu}}^{L}$, the other two vectors $\tilde{X}_{c(k)}^{L}, \tilde{X}_{c^{+}(k)}^{L}$ in $\mathcal{G}_{\Theta}$ (see Eq.(14)) should be understood as establishing the equivalence condition:

$$
\begin{aligned}
a_{\mu}(k) & \sim a_{\mu}(k)+i k_{\mu} c(k), \quad \phi(k) \sim \phi(k)+c(k) \\
a_{\mu}^{+}(k) & \sim a_{\mu}^{+}(k)-i k_{\mu} c^{+}(k), \quad \phi^{+}(k) \sim \phi^{+}(k)+c^{+}(k),
\end{aligned}
$$

where $c(k)$ and $c^{+}(k)$ are the corresponding integration parameters. The flow of the vector fields in Eq. (15) constitute the gauge transformations in the theory, and the relations (18) state simply that pure gauges do not contribute to the symplectic form $d \Theta / \mathcal{G}_{\Theta}$. The set of vector fields (15) is an ideal of $\mathcal{G}_{\Theta}$ and a horizontal (excluding the vertical $U(1)$-generator $\Xi$ ) ideal of the whole algebra $\tilde{\mathcal{G}}^{L}$ of $\tilde{G}$. For this subalgebra the right-invariant vector fields are proportional to the corresponding left ones and, therefore, the Noether invariants are zero. In fact, the proportionality functions provide a representation of the rest of the generators in the subalgebra $\mathcal{G}_{\Theta}$, more precisely, the Poincaré generators. These properties characterize the gauge subalgebra

$$
\mathcal{G}_{\text {gauge }} \equiv<\tilde{X}_{c(k)}^{L}, \tilde{X}_{c^{+}(k)}^{L}>
$$

of the theory [13].

Let $\mathcal{B}(\tilde{G})$ be the set of complex valued $T$-equivariant functions $\Psi$ on $\tilde{G}$, in the sense of principal bundle theory:

$$
\Psi(t * g)=D(t) \Psi(g), \quad \forall g \in \tilde{G}, \quad \forall t \in T
$$


where $D$ is a representation of $T$. The representation of $\tilde{G}$ on $\mathcal{B}(\tilde{G})$, generated by the right-invariant vector fields $\tilde{\mathcal{G}}^{R}=\left\{\tilde{X}^{R}\right\}$, is reducible. The reduction is achieved by means of the restriction imposed by a full polarization $\mathcal{P}$, that is, a maximal horizontal left subalgebra of $\tilde{\mathcal{G}}^{L}$ which contains the entire subalgebra $\mathcal{G}_{\Theta}$. This definition generalizes the analogous concept in Geometric Quantization [7] where no characteristic module exists (since all variables are symplectic). For the group $\tilde{G}$, two possible polarization subalgebras, corresponding with two possible representations, can be chosen depending on the value of $k^{2}$. From now on we shall distinguish between the cases $k^{2}=0$ and $k^{2}=m^{2} \neq 0$, placing each in a subsection. The former will lead to the Quantum Theory of Photons and the latter to the Quantum Proca Field, both requiring the imposition of constraints on the wave functions through $T$-equivariant conditions.

\section{$2.1 \tilde{G}\left(k^{2}=0\right)$ : Electromagnetic Field}

Firstly, we shall consider the case $k^{2}=0\left(\right.$ null mass $\left.^{\dagger}\right)$. For this case, the polarization subalgebra is:

$$
\mathcal{P}=<\tilde{X}_{x^{\mu}}^{L}, \tilde{X}_{c(k)}^{L}, \tilde{X}_{c^{+}(k)}^{L}, \tilde{X}_{a_{\mu}(k)}^{L}>\forall k
$$

As we have already mentioned, the wave functions in GAQ are the complex $T$-equivariant functions on the quantization group that are nullified by the (left) generators in the polarization. We shall perform the $U(1)$ part of the $T$ equivariance condition, impose the polarization equations and, separately, write the rest of the $T$-equivariance conditions, which then will appear as constraints, requiring further comments. The polarized $U(1)$-functions thus satisfy:

$$
\Psi(\zeta * g)=\zeta \Psi(g) \forall g \in \tilde{G}, \quad \tilde{X}^{L} \Psi=0, \quad \forall \tilde{X}^{L} \in \mathcal{P},
$$

or more explicitly:

$$
\begin{aligned}
\frac{\partial \Psi}{\partial x^{\mu}}-i \int \frac{d^{3} k}{2 k^{0}} k^{\mu}\left[a_{\nu}(k) \frac{\delta \Psi}{\delta a_{\nu}(k)}-a_{\nu}^{+}(k) \frac{\delta \Psi}{\delta a_{\nu}^{+}(k)}\right]- & =i \Psi \\
i \int \frac{d^{3} k}{2 k^{0}} k^{\mu}\left[\phi(k) \frac{\delta \Psi}{\delta \phi(k)}-\phi^{+}(k) \frac{\delta \Psi}{\delta \phi^{+}(k)}\right] & =0 \\
k_{\mu} \frac{\delta \Psi}{\delta a_{\mu}(k)}-i \frac{\delta \Psi}{\delta \phi(k)} & =0 \\
k_{\mu} \frac{\delta \Psi}{\delta a_{\mu}^{+}(k)}+i \frac{\delta \Psi}{\delta \phi^{+}(k)} & =0
\end{aligned}
$$

${ }^{\dagger}$ For this case the whole conformal group could be introduced, and the leaving the mass shell by the photon might be interpreted as a breaking of the conformal symmetry. 


$$
\frac{\delta \Psi}{\delta a_{\mu}(k)}-\frac{1}{2}\left[a^{+\mu}(k)+i \phi^{+}(k) k^{\mu}\right] \Psi=0
$$

with general solution:

$$
\begin{aligned}
\Psi\left(x^{\mu}, a_{\mu}, a_{\mu}^{+}, \phi, \phi^{+}, \zeta\right) & =\zeta \exp \left\{\frac { 1 } { 2 } \int \frac { d ^ { 3 } k ^ { \prime } } { 2 k ^ { \prime 0 } } \left(a_{\nu}^{+}\left(k^{\prime}\right) a^{\nu}\left(k^{\prime}\right)-i k^{\prime \nu} a_{\nu}^{+}\left(k^{\prime}\right) \phi\left(k^{\prime}\right)\right.\right. \\
& \left.\left.+i k^{\prime \nu} a_{\nu}\left(k^{\prime}\right) \phi^{+}\left(k^{\prime}\right)\right)\right\} \Phi\left(\left[a_{\mu}^{+}(k)+i k_{\mu} \phi^{+}(k)\right] e^{-i k x}\right) \\
& \equiv W \cdot \Phi\left(\left[a_{\mu}^{+}(k)+i k_{\mu} \phi^{+}(k)\right] e^{-i k x}\right)
\end{aligned}
$$

where $\Phi$ is an arbitrary power series on the argument $\left[a_{\mu}^{+}(k)+i k_{\mu} \phi^{+}(k)\right] e^{-i k x}$. The zero-order wave function, i.e. the vacuum, and the one-particle states of momentum $k$ are $\mid 0>\equiv W$ and $\mid a_{\mu}^{+}(k)>\equiv W \cdot\left[a_{\mu}^{+}(k)+i k_{\mu} \phi^{+}(k)\right] e^{-i k x}$, respectively.

Note that the Gaussian defining the vacuum contains the positive exponent $a_{0}^{+}\left(k^{\prime}\right) a^{0}\left(k^{\prime}\right)$ which could make the scalar product divergent. This breakdown will be avoided after the $T$-function conditions has been imposed to define the physical states of the theory. Let us explicitly show how the $T$-function conditions apply for this case. According to general settings (see ref. [5]), the structure group $T$ of the group $\tilde{G}$ is associated with constraints in the theory. They are imposed on the wave functions from the left as in eq. (20) or, in infinitesimal form:

$$
\tilde{X}^{R} \Psi_{\text {phys }}=d D\left(\tilde{X}^{R}\right) \Psi_{\text {phys }} \quad \forall \tilde{X}^{R} \in \mathcal{T} \equiv<\Xi, \tilde{X}_{\phi(k)}^{R}, \tilde{X}_{\phi^{+}(k)}^{R}>
$$

where $d D$ is the "differential" of the group representation $D$ in (20) characterizing the representation. We have already imposed the $U(1)$ part, i.e. that corresponding to the $U(1)$-function condition $\Xi \Psi=i \Psi$. For the rest, we take the trivial representation $d D=0$, and we consider the action of $\tilde{X}_{\phi(k)}^{R}$ and $\tilde{X}_{\phi^{+}(k)}^{R}$ on an arbitrary combination of one-particle states $\epsilon^{\mu}(k) \mid a_{\mu}^{+}(k)>$. Then we get:

$$
\begin{aligned}
\tilde{X}_{\phi^{+}(k)}^{R} \epsilon^{\mu}(k) \mid a_{\mu}^{+}(k)>= & =\Rightarrow \epsilon^{\mu}(k) k_{\mu}=0 \\
\tilde{X}_{\phi(k)}^{R} \Psi_{\text {phys }} & =0 \Rightarrow k^{\mu} a_{\mu}^{+}(k) \Psi_{\text {phys }}=0 .
\end{aligned}
$$

The first condition establishes that physical states must contain the same amount of longitudinal photons as time-like ones for all $k$. This condition also guarantees that physical states have positive (or null) norm, since $-\epsilon^{\mu+}(k) \epsilon_{\mu}(k) \geq 0$, and it recovers the well-known Gupta-Bleuler condition [8, 9]. The second condition in (26) eliminates the null vectors (null-norm vectors) from the theory. It simply states that all the zero-norm vectors, created by the action of $\tilde{X}_{\phi(k)}^{R}$, are equivalent to zero. In other words, the physical wave functions $\Psi_{\text {phys }}$ have support only on the "surface" $k^{\mu} a_{\mu}^{+}(k)=0$, thus avoiding the above-mentioned breakdown in the scalar product due to the positive exponent $a_{0}^{+}\left(k^{\prime}\right) a^{0}\left(k^{\prime}\right)$ in the Gaussian defining the vacuum. That is, one has to integrate only on physical degrees of 
freedom (transversal components) in calculating a scalar product. In the standard approach (see e.g. [10]), it is usally preferred to allow the null vectors to circulate in the system or to introduce an equivalence relation ("two states differing in a null vector are equivalents") and select an equivalence class. This way of proceeding is justified by the fact that the mean values of physical operators prove to be independent of the chosen equivalence class (see the Sect. 3 for a thorough discussion of these facts).

Since the $T$-equivariance condition is imposed by means of right generators, it is obvious that not all the operators (right generators) will preserve the space of $T$-equivariant states (physical states). In the present case the operators (named good in the general approach, see Ref. [5]) preserving this space are:

$$
\mathcal{G}_{\text {good }}=<\epsilon_{\mu}(k) \tilde{X}_{a_{\mu}(k)}^{R}, \epsilon_{\mu}^{+}(k) \tilde{X}_{a_{\mu}^{+}(k)}^{R}, \tilde{X}_{x^{\mu}}^{R}>\forall k
$$

where the factors $\epsilon^{\mu}(k)$ are restricted by the two conditions (26) that is, they verify $\epsilon^{\mu}(k) k_{\mu}=0$ and $-\epsilon^{\mu+}(k) \epsilon_{\mu}(k)>0$. Thus, they project on transversal components and keep two field degrees of freedom out of the original four field degrees of freedom. The good operators in (27) behave as the creation and annihilation operators of transversal states:

$$
\epsilon_{\mu}(k) \hat{a}^{+\mu}(k) \equiv \epsilon_{\mu}(k) \tilde{X}_{a_{\mu}(k)}^{R}, \epsilon_{\mu}^{+}(k) \hat{a}^{\mu}(k) \equiv \epsilon_{\mu}^{+}(k) \tilde{X}_{a_{\mu}^{+}(k)}^{R},
$$

respectively, and the Poincaré operators,

$$
P_{\mu} \equiv i \tilde{X}_{x^{\mu}}^{R}, M_{\mu \nu} \equiv i \tilde{X}_{\Lambda^{\mu \nu}}^{R}
$$

(when the Lorentz transformations $\Lambda$ are kept) declare, in particular, that the electromagnetic field carries helicity \pm 1 . They close a Lie subalgebra of the original one, and constitute the physical operators of the theory.

\section{$2.2 \tilde{G}\left(k^{2} \neq 0\right)$ : Proca Field}

As mentioned above, a remarkable characteristic of the quantizing group (10) is that it accomplishes the quantization of both the electromagnetic and Proca fields in a unified way. The term in the cocycle proportional to $k^{2}$ causes the photon to acquire mass at the same time that it breaks the conformal invariance of the theory. To see this, let us show how it is possible, for this case, to decouple the gauge field by means of a transformation which diagonalizes the cocycle. In fact, the combinations:

$$
\tilde{X}_{b_{\mu}(k)}^{L} \equiv \tilde{X}_{a_{\mu}(k)}^{L}-i \frac{k^{\mu}}{k^{2}} \tilde{X}_{\phi(k)}^{L}, \quad \tilde{X}_{b_{\mu}^{+}(k)}^{L} \equiv \tilde{X}_{a_{\mu}^{+}(k)}^{L}+i \frac{k^{\mu}}{k^{2}} \tilde{X}_{\phi^{+}(k)}^{L}
$$

together with $\tilde{X}_{\phi(k)}^{L}$ and $\tilde{X}_{\phi^{+}(k)}^{L}$ close the Lie algebra: 


$$
\begin{aligned}
& {\left[\tilde{X}_{b_{\mu}^{+}(k)}^{L}, \tilde{X}_{b_{\nu}\left(k^{\prime}\right)}^{L}\right]=i M^{\mu \nu}(k) \Delta_{k k^{\prime}} \Xi, \quad\left[\tilde{X}_{\phi^{+}(k)}^{L}, \tilde{X}_{\phi\left(k^{\prime}\right)}^{L}\right]=i k^{2} \Delta_{k k^{\prime}} \Xi} \\
& {\left[\tilde{X}_{b_{\mu}(k)}^{L}, \tilde{X}_{\phi\left(k^{\prime}\right), \phi^{+}\left(k^{\prime}\right)}^{L}\right]=0, \quad\left[\tilde{X}_{b_{\mu}^{+}(k)}^{L}, \tilde{X}_{\phi\left(k^{\prime}\right), \phi^{+}\left(k^{\prime}\right)}^{L}\right]=0}
\end{aligned}
$$

where $M^{\mu \nu}(k) \equiv g^{\mu \nu}-\frac{k^{\mu} k^{\nu}}{k^{2}}$. The two first commutators are the Proca-like and the real Klein Gordon-like ones respectively, and the others simply state that those two fields are decoupled.

For this case, the polarization subalgebra is made of the following left generators:

$$
\mathcal{P}=<\tilde{X}_{x^{\mu}}^{L}, \tilde{X}_{c(k)}^{L}, \tilde{X}_{c^{+}(k)}^{L}, \tilde{X}_{b_{\mu}(k)}^{L}, \tilde{X}_{\phi^{+}(k)}^{L}>\forall k
$$

The integration of the polarization conditions essentially follows that of Ref. [11], and, together with the $U(1)$-function condition, leads to:

$$
\begin{aligned}
\Psi= & \zeta \exp \left\{-\frac{1}{2} \int \frac{d^{3} k^{\prime}}{2 k^{\prime 0}}\left[\sum_{i=1}^{3} \alpha^{i}\left(k^{\prime}\right) \alpha^{+i}\left(k^{\prime}\right)+k^{2} \chi\left(k^{\prime}\right) \chi^{+}\left(k^{\prime}\right)\right]\right\} . \\
& \Phi\left(\left[\alpha^{+j}(k) e^{-i k x}\right],\left[\chi(k) e^{i k x}\right]\right) \equiv W \cdot \Phi
\end{aligned}
$$

where $\Phi$ is an arbitrary function of its arguments; we have defined $\chi(k) \equiv \phi(k)+$ $i \frac{k^{\mu}}{k^{2}} a_{\mu}(k)$, and the coefficients $\alpha^{i}(k), \alpha^{+i}(k)$ are the transverse part of

$$
a_{\mu}(k)=\sum_{\beta=0}^{3} \alpha^{\beta}(k) \epsilon_{\mu}^{\beta}(k), \quad a_{\mu}^{+}(k)=\sum_{\beta=0}^{3} \alpha^{+\beta}(k) \epsilon_{\mu}^{\beta}(k),
$$

$\epsilon_{\mu}^{\beta}(k)$ being a tetrad defined by

$$
\begin{array}{ll}
g^{\mu \nu} \epsilon_{\mu}^{\beta}(k) \epsilon_{\nu}^{\sigma}(k)=g^{\beta \sigma} & k^{\mu} \epsilon_{\mu}^{i}(k)=0, \quad i=1,2,3 \\
\epsilon_{\mu}^{0}(k)=k_{\mu} / k & \sum_{i=1}^{3} \epsilon_{\mu}^{i}(k) \epsilon_{\nu}^{i}(k)=-M_{\mu \nu}(k) .
\end{array}
$$

Note that we have four field degrees of freedom. For this case, the structure group $T$ is itself a central extension and, according to general settings (see Ref. [5]), only a subgroup $T_{B}=T_{p} \cup U(1)$ of $T$ can be consistently imposed as $T$ equivariant condition. $T_{p}$ is a polarization subgroup of $T$ which we can choose to be $T_{p}=<\tilde{X}_{\phi(k)}^{R}>$. We have already imposed the $U(1)$-function condition, so that the rest of the $T_{B}$-function condition states that

$$
\tilde{X}_{\phi(k)}^{R} \Psi=0 \Rightarrow \frac{\delta \Phi}{\delta \chi(k)}=0 \quad \forall k,
$$

that is, the arbitrary function $\Phi$ does not depend on the $\chi$ variable. On the other hand, if we chose $T_{p}=<\tilde{X}_{\phi^{+}(k)}^{R}>$ as the polarization subgroup of $T$, we would obtain:

$$
\tilde{X}_{\phi^{+}(k)}^{R} \Psi=0 \Rightarrow \chi(k) \Psi=0 \forall k
$$


which simply states that the wave function $\Psi$ has support only at the values

$$
\chi(k)=0 \Rightarrow k \phi(k)=-i \alpha^{0}(k) \forall k .
$$

This vaguely resembles the Higgs Mechanism, where the Goldston bosons are eliminated from the theory by making use of the gauge freedom.

In any case, the rest of the wave function, i.e.

$$
\Psi=W \cdot \Phi\left(\left[\alpha^{+j}(k) e^{-i k x}\right]\right),
$$

is exactly the Proca quantum wave function (see [11] for more details). The non-trivial good operators for this case are:

$$
\mathcal{G}_{\text {good }}=<\epsilon_{\mu}^{j}(k) \tilde{X}_{b_{\mu}(k)}^{R}, \epsilon_{\mu}^{j}(k) \tilde{X}_{b_{\mu}^{+}(k)}^{R}, \tilde{X}_{x^{\mu}}^{R}>, \quad j=1,2,3
$$

where $\hat{\alpha}^{+j}(k) \equiv \epsilon_{\mu}^{j}(k) \tilde{X}_{b_{\mu}(k)}^{R}$ and $\hat{\alpha}^{j}(k) \equiv \epsilon_{\mu}^{j}(k) \tilde{X}_{b_{\mu}^{+}(k)}^{R}$ are the creation and annihilation operators of transversal components, respectively. The whole Poincaré subgroup is also good. The longitudinal components $\epsilon_{\mu}^{0}(k) \tilde{X}_{b_{\mu}(k)}^{R}$ and $\epsilon_{\mu}^{0}(k) \tilde{X}_{b_{\mu}^{+}(k)}^{R}$ prove to be identically zero on polarized functions (33); even more, they are respectively proportional to the gauge generators $\tilde{X}_{c(k)}^{L}, \tilde{X}_{c^{+}(k)}^{L}$ and, therefore, they also have a null Noether invariant.

\section{Comments and outlook}

The main achievement of the present paper is the quantization of both the electromagnetic and Proca fields within a unified, general scheme of quantization based on a group structure, which is specially suited for dealing with constrained systems. To achieve this goal, the concept of gauge symmetry for the electromagnetic field has been revisited, giving rise to a subtle distinction between the constraint and the gauge subgroups. Gauge symmetries are associated with horizontal ideals of the general symmetry group, the right generators of which prove to have null Noether invariants and are, therefore, proportional to the corresponding left generators. On the other hand, the constraint subgroup is essentially the structure group of the principal fibre bundle $\tilde{G}$, which is used as the starting point for our Group Approach to Quantization.

One striking result of the present mechanism is the persistence of the gauge symmetry in the massive case. In this case the possibility exists also of not imposing the constraints, thus allowing for one extra (scalar) massive field with "negative energy" and decoupled from the Proca field. For the non-abelian theory the situation seems to be less trivial and the connection with some sort of symmetry-breaking mechanism deserves a further study.

With respect to constraints in the specific $k^{2}=0$ case, we wish to point out that, in principle, and from an algebraic viewpoint, both generators $\tilde{X}_{\phi(k)}^{R}$ 
and $\tilde{X}_{\phi^{+}(k)}^{R}$ can be compatibily imposed since, unlike in the $k^{2} \neq 0$ case, their commutator is zero. It is thoroughly argued in the literature that such a set of constraints cannot be simultaneously imposed because otherwise the commutator $\left[k^{\mu} \hat{a}_{\mu}^{+}(k), \hat{a}_{\nu}\left(k^{\prime}\right)\right]=k_{\nu} \Delta_{k k^{\prime}}$ should imply that the state $\hat{a}_{\nu}\left(k^{\prime}\right) \mid$ physical $>$ is not in general a physical state; even more, not even the vacuum could satisfy the identity $k^{\mu} \hat{a}_{\mu}^{+}(k) \mid 0>=0$. However, in our scheme, only a subgroup of operators, $\mathcal{G}_{\text {good }}$, can be consistently quantized, in fact, the transversal components of the electromagnetic field and the Poincaré generators. In this way, our theory is free from ghosts and null vectors. Note that, when the photon is off shell, only one of the two $\tilde{X}_{\phi(k)}^{R}$ or $\tilde{X}_{\phi^{+}(k)}^{R}$ operators can be consistently imposed as constraint; this "obstruction" has now an algebraic origin: a couple of canonically conjugated variables cannot be simultaneously set to zero.

For completion, and in an attempt to extend this formalism to non-abelian gauge theories, we express the group law (10) directly in configuration space (see [12]), where no knowledge of the solutions of the classical equations of motion is required to obtain non-trivial conclusions about the corresponding quantum theory. Although the quantization of the non-abelian gauge theories from this new approach is beyond the objectives of this paper, the writing of the group law (10) in configuration space may shed some new light on the non-abelian case, which deserves further study.

The abovementioned group law is (we discard, for simplicity, the Poincaré subgroup):

$$
\begin{aligned}
A_{\mu}^{\prime \prime}(x)= & A_{\mu}^{\prime}(x)+A_{\mu}(x) \\
\varphi^{\prime \prime}(x)= & \varphi^{\prime}(x)+\varphi(x) \\
\zeta^{\prime \prime}= & \zeta^{\prime} \zeta^{\frac{i}{2} \int_{\Sigma} d \sigma_{\mu}(x) J^{\mu}\left(g^{\prime}, g\right)(x)} \\
J^{\mu}\left(g^{\prime}, g\right)(x)= & A_{\rho}^{\prime}(x) \overleftrightarrow{\partial^{\mu}} A^{\rho}(x)+\varphi^{\prime}(x) \overleftrightarrow{\partial^{\mu}}\left(\partial_{\rho} A^{\rho}(x)\right)-\varphi(x) \overleftrightarrow{\partial^{\mu}}\left(\partial_{\rho} A^{\prime \rho}(x)\right) \\
& +m^{2} \varphi^{\prime}(x) \overleftrightarrow{\partial^{\mu}} \varphi(x)
\end{aligned}
$$

where $\varphi^{\prime}(x) \overleftrightarrow{\partial^{\mu}} \varphi(x) \equiv \varphi^{\prime}(x) \partial^{\mu} \varphi(x)-\varphi(x) \partial^{\mu} \varphi^{\prime}(x)$ and so on. The parameters $A_{\mu}(x)$ and $\varphi(x)$ have to satisfy the equations

$$
\begin{aligned}
\partial_{\mu} F^{\mu \nu}(x)+\partial^{\nu}\left(\partial_{\rho} A^{\rho}(x)\right)+m^{2} A^{\nu}(x) \equiv\left[\partial_{\mu} \partial^{\mu}+m^{2}\right] A^{\nu}(x) & =0 \\
{\left[\partial_{\mu} \partial^{\mu}+m^{2}\right] \varphi(x) } & =0
\end{aligned}
$$

for the current $J^{\mu}\left(g^{\prime}, g\right)(x)$ to be conserved $\left(\partial_{\mu} J^{\mu}=0\right)$, so that the integral defining the cocycle does not depend on the chosen space-like hypersurface $\Sigma$ (see [12 for further details).

As already mentioned, the group law (41) suggests a revision of the concept of gauge transformation for the vector potential $A_{\mu}(x)$. According to our scheme, the action of the $U(1)_{l o c}$ subgroup in the group law (41), for $m=0$, leaves the 
vector potential unchanged, although it changes the central parameter $\zeta$ (and, accordingly, the phase of the wave function). More explicity:

$$
\varphi(x) \rightarrow \varphi(x)+\varphi^{\prime}(x), \quad A_{\mu}(x) \rightarrow A_{\mu}(x), \quad \zeta \rightarrow \zeta e^{\frac{i}{2} \int_{\Sigma} d_{\sigma_{\mu}}(x) \varphi^{\prime}(x) \overleftrightarrow{\partial \vec{\partial}^{\prime}}\left(\partial_{\rho} A^{\rho}(x)\right)}
$$

However, the standard transformation (11) is regained as the trajectories associated with the vector fields in the gauge subalgebra (19). The same revision applies for the non-abelian case, where the situation seems to be a little more subtle.

Finally, we must mention that the analysis in this paper is, in fact, a particular case of a more general one in which a parameter $\lambda$ (see (6)) should be kept arbitrary.

\section{Acknowledgments}

We all wish to thank J. Guerrero for valuable discussions. M. C. and M. N. are grateful to the Spanish M.E.C. for a F.P.I. and postdoctoral F.P.U. grant, respectively, and M. N. to the Imperial College for its hospitality. 


\section{References}

[1] I. Bialynicki-Birula and Z. Bialynicka-Birula, Quantum Electrodynamics, Pergamon Press 1975.

[2] R. Jackiw, Topological investigations of quantized gauge theories, in Current algebra and anomalies, S.B. Treiman et al., eds. World Scientific (1985).

[3] V. Aldaya and J. Navarro-Salas, Phys. Lett. B274, 79 (1992)

[4] V. Aldaya and J. de Azcárraga, J. Math. Phys. 23, 1297 (1982)

[5] V. Aldaya, J. Navarro-Salas and A. Ramírez, Comm. Math. Phys. 121, 541 (1989)

[6] V. Aldaya, R. Loll and J. Navarro-Salas, Phys. Lett. B237, 392 (1989)

[7] B. Kostant, Quantization and Unitary Representations (Lecture Notes in Mathematics 170), Berlin:Springer, (1970).

[8] S.N. Gupta, Proc. Phys. Soc. Lon. A63, 681, (1950).

[9] K. Bleuler, Helv. Phys. Acta 23, 567, (1950).

[10] C. Itzykson and J.B. Zuber, Quantum Field Theory, McGraw-Hill 1985.

[11] V. Aldaya, J.A. de Azcárraga and S. García, J. Phys. A21, 4265 (1988).

[12] M. Navarro, V. Aldaya and M. Calixto, J. Math. Phys, 37, 206 (1996).

[13] M. Navarro, V. Aldaya and M. Calixto, Group Quantization on Configuration Space: Gauge Symmetries and Linear Fields, preprint Imperial-TP/95$96 / 12$. 